\title{
The Importance of Diversity to Public Health
}

David SATCher, MD, PhD

I have always considered veterinary medicine to be an important part of public health, and during my career I have had the opportunity to work with many outstanding veterinarians who have contributed much to public health practice. But I think there are ways we can work together to improve health in this country.

As the former U.S. Surgeon General, I talked about the need for a balanced health system-a system that balances health promotion, disease prevention, early detection, and universal access. It is the collective efforts of a society that create the environmental conditions in which people can be healthy. There are many implications in that definition for our health-care system, veterinary medicine, and the collective efforts to create good environmental conditions, including the social environment.

Only $2 \%$ of today's veterinary school students are black and $5 \%$ or fewer are Hispanic. I think we need to create the kind of environment in which people who are training to be veterinarians, or learning to be public health leaders, learn in a racially and ethnically diverse environment so they know how to handle diversity in our society. I believe there are three critical reasons why diversity is important.

For one, our children may not be getting the opportunity to develop their skills and their talents. For another, diversity is an integral part of our environment. Diversity, or the lack of it, defines our environment. If only $2 \%$ of today's veterinary students are black, it means that many veterinary students will never get to know a black student. I think one of the ways that people learn cultural diversity is by getting to know people from different racial/ethnic backgrounds. They get to know their life history. They get to know what makes them tick. They get to know their strengths and weaknesses. Finally, diversity can help eliminate health disparities. This is one of the two goals provided in Healthy People 2010 that is composed of a set of health objectives for the nation. Clearly, one of the critical issues in eliminating disparities is diversity in the health professions and in all areas of public health, including veterinary health.

There is a critical need for more diversity to focus on universal problems, to get providers in communities where they are needed, and to create a system that supports those needs. Diversity is important because it defines the parameters of opportunities for our children; it enriches the lives of our future professionals; and because if we are going to achieve the goal of eliminating disparities in health, we'll need a diverse group of health professionals to accomplish it.

David Satcher

Center of Excellence on Health Disparities, Morehouse School of Medicine Atlanta, GA 